\title{
LÍMITES DE DETECCIÓN DE ALGUNOS MÉTODOS DE PRUEBA PARA ADULTERANTES E INHIBIDORES EN LECHE
}

\author{
Limits of detection for analytical methods to detect adulterants and inhibitors in \\ milk.
}

\begin{abstract}
Mario Noa- Pérez ${ }^{1}$, Miriam Cortés- Marín ${ }^{2}$, Patricia Landeros- Ramírez ${ }^{1}$, Zoila GómezCruz $^{1}$, Mario Real- Navarro ${ }^{1}$, Ramón Reynoso- Orozco ${ }^{1}$, Teresa de Jesús Jaime Ornelas ${ }^{1}$ y Carlos Juárez-Woo ${ }^{1}$
\end{abstract}

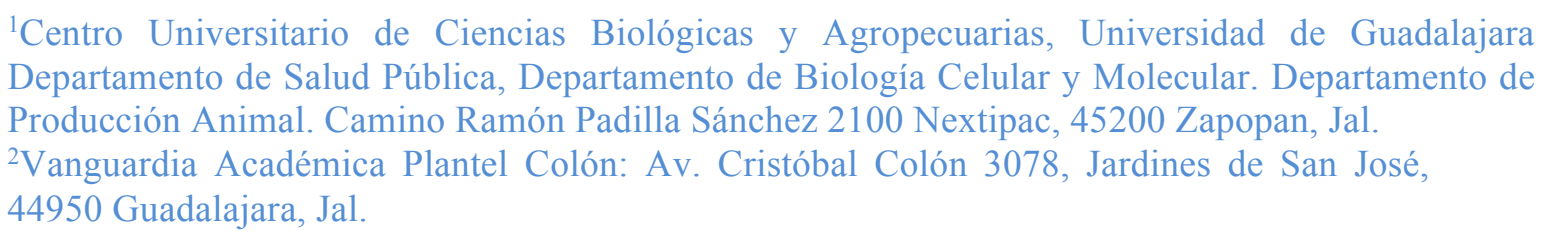

*Autor de correspondencia: mario.noa@academicos.udg.mx

\section{Resumen}

La legislación mexicana considera adulterado un producto cuando se encubran defectos en su proceso o en la calidad sanitaria de las materias primas utilizadas. En leche, los principales adulterantes incorporados incluyen aquellos adicionados para incrementar el volumen de la leche y los que enmascaran la acidificación. Además, la leche también debe ser negativa a la presencia de inhibidores de crecimiento microbiano, ya sean antibióticos, o químicos. Aunque está claramente establecida la prohibición de su presencia, los límites de detección (LD) de almidón y féculas, sacarosa, gelatina, desinfectantes clorados, oxidantes, de amonio cuaternario y 14 inhibidores de crecimiento microbiano de mayor uso, no están reflejados en la regulación mexicana vigente para leche. Por ello, se implementaron metodologías analíticas químicas cualitativas para los indicadores de selectividad y límite de detección de algunos adulterantes, así como la prueba de inhibición del yogurt para inhibidores (antimicrobianos y desinfectantes). El control negativo para los indicadores fue leche descremada en polvo de calidad microbiológica y para la prueba de yogurt el cultivo comercial YO-MIX

TM. Mediante métodos químicos se obtuvieron los LD para almidón y féculas comerciales $(125$ y $250 \mathrm{mg} / \mathrm{kg})$, gelatina $(250 \mathrm{mg} / \mathrm{kg})$, peróxido de hidrógeno (6 $\mathrm{mg} / \mathrm{kg})$, hipoclorito de sodio $(25 \mathrm{mg} / \mathrm{kg})$, Cloruro de benzalconio $(12 \mathrm{mg} / \mathrm{kg}), \quad \mathrm{N}$-Alquil $(\mathrm{C} 12$, 16)- N,N-dimetil- Nbencilamonio $(6 \mathrm{mg} / \mathrm{kg})$. La prueba del yogurt mostró una sensibilidad para hipoclorito de sodio (25 $\mathrm{mg} / \mathrm{kg}$ ), compuestos de amonio cuaternario $\quad(60 \quad \mathrm{mg} / \mathrm{kg}), \quad$ y $\quad$ los oxidantes no fueron detectables, pero fue suficientemente sensible para los 14 antimicrobianos ensayados a sus LMR. En este trabajo se pudieron detectar cualitativamente los LD de otros parámetros de calidad no establecidos aun en la normativa mexicana vigente para leche. Se recomienda efectuar ambos tipos de ensayos, químicos y del yogurt de forma simultánea, para determinar la mayor cantidad de adulterantes e inhibidores a los niveles de interés. 
Palabras claves: leche, adulterantes, inhibidores, límites de detección, métodos analíticos.

\section{Abstract}

The Mexican Regulations on milk classifies a product as adulterated when it has either undergone treatment that conceals its alteration and/or defects in its process or in the sanitary quality of the raw materials, among other criteria. The principal, most widely known adulterants incorporated into milk, include those added to increase milk volume and mask acidification. Milk should also test negative for the presence of inhibitors, e.g. antibiotics or disinfectants. The objective of this work was to determine the LOD for starches, sucrose, gelatin, chlorinated disinfectants; oxidants, quaternary ammonium and 14 most widely used microbial growth inhibitors in milk. This is because although the prohibition of these substances is clearly established, the detection limits (LOD) are not declared in the current regulation. With this finality, qualitative chemical analytical methodologies for detection of adulterants described in the Mexican Standards were implemented, as well as for the Yogurt Inhibition Test. Microbiological quality skim milk powder was used as a negative control, and the commercial culture YO-MIX TM was used for the Yogurt Inhibition Test. The following LOD were obtained by chemical methods for starches (125 and $250 \mathrm{mg} / \mathrm{kg})$, gelatin $(250 \mathrm{mg} / \mathrm{kg})$, hydrogen peroxide $(6 \mathrm{mg} / \mathrm{kg})$, sodium hypochlorite $(25 \mathrm{mg} / \mathrm{kg})$, benzalkonium chloride $(12 \mathrm{mg} / \mathrm{kg}), \mathrm{N}-A$ lkyl $(\mathrm{C} 12,16)$ $\mathrm{N}, \mathrm{N}$-dimethyl-N- benzylammonium (6 $\mathrm{mg} / \mathrm{kg}$ ). The yogurt test showed higher LOD for the disinfectants: $25,000 \mathrm{mg} / \mathrm{kg}$ for sodium hypochlorite and $60 \mathrm{mg} / \mathrm{kg}$ for the quaternary ammonium compounds. On the other hand, although oxidants were not detectable the yogurt test was sufficiently sensitive to detect the Maximum Residue Limits (MRLs) for the 14 antimicrobials tested. Carrying out both types of tests, chemical and yogurt, to determine disinfectants and inhibitors at the levels of interest in milk is recommended.

Keywords: milk, adulterants, inhibitors, detection limits, analytical methods.

\section{Introducción}

Un producto se considera adulterado cuando su naturaleza y composición no correspondan a aquéllas con que se etiquete, anuncie, expenda, suministre o cuando no corresponda a las especificaciones de su autorización, o haya sufrido tratamiento que disimule su alteración, se encubran defectos en su proceso o en la calidad sanitaria de las materias primas utilizadas (Ley General de Salud, 1984). Por lo tanto, la incorporación de sólidos no lácteos en la leche fluida, se considera adulteración.

Los principales adulterantes conocidos que son incorporados a la leche, y que constituyen fraudes para el consumidor, se dividen en tres grupos: los que son adicionados directamente a la leche para incrementar el volumen de leche de forma fraudulenta (agua, sacarosa, almidones, gelatina, etc.), los que tratan de enmascarar defectos en la calidad de la leche, como la acidificación (álcalis, formaldehido, agentes oxidantes y otros agentes microbianos), y aquellos que sustituyen componentes propios de la leche por otros de menor costo, como grasa no láctea o suero de quesería (Vega, Pérez \& Pinto, 1999).

En leche, los agentes antimicrobianos o inhibidores bacterianos incluyen tanto antibióticos como desinfectantes. Entre éstos últimos, se pueden encontrar 
compuestos químicos derivados clorados, sales cuaternarias de amonio, oxidantes o formaldehido. Ambos grupos de sustancias pueden tener la función de adulterar la leche para enmascarar su mala calidad bacteriológica. La normatividad mexicana referida tanto a leche cruda de vaca como pasteurizada, establece que la presencia de dichos inhibidores bacterianos debe ser negativa (NOM-243-SSA1-2010, NMX-F-700COFOCALEC-2012).

Sin embargo, estudios anteriores han demostrado la presencia de antimicrobianos en leche cruda destinada a la industria procesadora (Noa et al, 2019, Noa-Lima, Noa, González, Landeros y Reyes, 2009; Solares, 2019). Los agentes antimicrobianos se encuentran entre los medicamentos más comúnmente utilizados en medicina veterinaria. Desafortunadamente el uso generalizado de estas sustancias tuvo como consecuencia la aparición de agentes patógenos resistentes a los antimicrobianos. Esta situación ha alimentado la cada vez mayor necesidad de nuevos medicamentos, aunque el ritmo del desarrollo de los medicamentos antimicrobianos ha disminuido dramáticamente en los últimos años. Lo anterior se debe principalmente a que se cree que reducir el uso inapropiado de antibióticos es la mejor manera para controlar la resistencia microbiana (Goodman y Gilman's, 2008); y a que las grandes empresas ya no quieren producirlos debido a que su proceso es muy costoso e implica varios años de estudios.

En este sentido, la OMS ha implementado diversas iniciativas para combatir la resistencia a antimicrobianos, como el Sistema Mundial de Vigilancia de la Resistencia a los Antimicrobianos (GLASS por sus siglas en inglés) el cual, apoya y promueve la adopción de un enfoque normalizado para la recopilación, el análisis y publicación de datos sobre la resistencia a los antimicrobianos a nivel mundial (OMS, 2020).

Para apoyar esta iniciativa, es importante contar con métodos de pruebas o procedimientos analíticos utilizados en el laboratorio, que permitan comprobar que un producto satisface las especificaciones que establece la normatividad vigente y que en el caso de México, para la leche se refiere a la NOM-155-SCFI-2012.

Aunque está claramente establecida la prohibición de la presencia de antimicrobianos en leche, en muchos casos, sus límites de detección (LD) no están reflejados en la normatividad. Debido a lo anterior, el objetivo del presente trabajo fue determinar los límites de detección para algunos adulterantes e inhibidores de crecimiento microbiano en leche, utilizando las metodologías analíticas recomendadas por la normatividad mexicana vigente o en su defecto, por métodos seleccionados de la bibliografía internacional.

\section{Materiales y métodos}

Se implementaron las metodologías analíticas cualitativas de tipo químico para la detección de adulterantes e inhibidores descritos en la NOM-243SSA1-2010 para las siguientes sustancias: derivados clorados, agentes oxidantes y compuestos de amonio cuaternario. Para la determinación de otros adulterantes como almidón, sacarosa y gelatina (no incluidos en la norma oficial mexicana antes mencionada), se utilizaron los procedimientos analíticos descritos en los Métodos de Análisis de la AOAC (2006) y de la Unión Europea (CEE, 2008). La prueba de inhibición de yogurt (NMX-F719-COFOCALEC-2008), se utilizó para la detección de antimicrobianos 
(inhibidores y desinfectantes). Los métodos de prueba utilizados se muestran en la Tabla 1.

Tabla 1. Métodos de prueba químicos utilizados para la detección de adulterantes e inhibidores en leche.

\begin{tabular}{|c|c|c|}
\hline Adulterante o inhibidor & Método de prueba & Referencia \\
\hline Almidón y féculas & Prueba del lugol & CEE, 2008 \\
\hline Sacarosa & Reacción de Seliwanoff & Artica, 2016 \\
\hline Gelatina & & AOAC, 2006 \\
\hline $\begin{array}{l}\text { Derivados clorados } \\
\text { Agentes oxidantes }\end{array}$ & \multirow{2}{*}{ Colorimétrico } & \multirow[b]{2}{*}{ NOM-243-SSA1- 2010} \\
\hline $\begin{array}{l}\text { Compuestos de amonio } \\
\text { cuaternario }\end{array}$ & & \\
\hline Inhibidores & $\begin{array}{l}\text { Prueba de inhibición de } \\
\text { yogurt }\end{array}$ & $\begin{array}{l}\text { NMX-F-719- } \\
\text { COFOCALEC-2008 }\end{array}$ \\
\hline
\end{tabular}

Procedimientos para la determinación de los $L D$ de adulterantes e inhibidores.

Considerando que los métodos de prueba implementados en el presente estudio son de carácter cualitativo, sólo se tuvieron en cuenta los indicadores de selectividad y límite de detección, según Thompson, Ellison y Wood (2002). Para ambos indicadores se utilizó como control negativo, leche descremada en polvo de calidad microbiológica Difco ${ }^{\mathrm{TM}}$ Skim Milk reconstituida en agua destilada estéril en una relación de $10 \%$ de sólidos (p/v).

Los LD se determinaron contaminando una leche control negativa (Difco ${ }^{\mathrm{TM}}$ Skim Milk) con cada sustancia a ensayar. Para ello, se adicionó a una muestra de leche control negativo un volumen aproximado del $1 \%$ de cada adulterante o inhibidor a ensayar, hasta obtener un resultado positivo, o sea, el Límite de Detección (LD). El valor obtenido de LD se repitió 10 veces para cada sustancia ensayada.

Para determinar la selectividad de cada método de prueba, se ensayaron diferentes productos, incluyendo formulaciones comerciales que contenían el adulterante en cuestión, cuya presencia fuera probable encontrar en leche.

Para cada uno de los antibióticos y desinfectantes se determinaron además, los límites de detección (LD) positivos y negativos para la prueba del yogurt. Por ello se estableció la menor concentración a la cual la prueba resulta positiva, así como la mayor concentración a la que todos los resultados fueron negativos; elaborando 10 repeticiones de cada valor. La cepa de yogurt utilizada fue YO-MIX TM (DANISCO, Francia).

Los métodos de análisis aplicados en todos los casos en el presente trabajo, se conocen como métodos de monitoreo o screening (o criba) y se validan con la determinación de los LD seleccionados (concentración más baja en la cual se ha detectado un analito) en al menos el 95\% de 5 muestras analizadas (Codex Alimentarius, 2017).

Determinación de almidón y féculas 
Para ensayar el método se utilizaron almidón grado analítico y fécula de maíz de calidad comercial aplicando la Prueba de Lugol (CEE, 2008). Este método de prueba no está incluido en la normatividad mexicana vigente para leche, y detecta el almidón en la leche en polvo desnaturalizada. Se refiere que el LD del método fue de $0.05 \mathrm{~g}$ de almidón por $100 \mathrm{~g}$ de muestra $(500 \mathrm{mg} / \mathrm{kg})$.

\section{Sacarosa}

En la leche pueden encontrarse moléculas de glucosa provenientes de la hidrólisis de la lactosa, pero debe estar exenta de fructosa. Por lo tanto, los métodos analíticos para detectar sacarosa se fundamentan en la detección de fructosa. Para ensayar el método se aplicó la Reacción de Seliwanoff (Artica, 2016), utilizando como estándar sacarosa de grado analítico.

\section{Gelatina}

La presencia de gelatina se determinó por reacción de la leche con ácido pícrico (AOAC, 2006). Para ensayar el método se utilizó grenetina de grado comercial.

Derivados clorados (hipoclorito de sodio)

La presencia de cloro en leche determinó por contacto con yoduro de potasio y una solución de almidón (NOM-243-SSA1-2010). Para ello se utilizó hipoclorito de sodio de grado comercial.

Agentes oxidantes (peróxido de hidrógeno)

La presencia de agentes oxidantes en leche se determinó por contacto con pentóxido de vanadio en presencia de ácido sulfúrico al 6\% (v/v) (NOM-243SSA1-2010) utilizando peróxido de hidrógeno de calidad comercial como agente oxidante.

Compuestos de amonio cuaternario ( $N$ Alquil $\quad[C 12,16]-N, N$-dimetil- $\quad \mathrm{N}$ bencilamonio y cloruro de benzalconio)

La presencia de compuestos cuaternarios de amonio se determinó al reaccionar el ion cuaternario de amonio eventualmente presente en la leche, en medio alcalino $y$ en presencia del indicador anaranjado de metilo (NOM243-SSA1-2010); empleando cloruro de benzalconio grado reactivo y $\mathrm{N}$-Alquil $(\mathrm{C} 12,16)-\mathrm{N}, \mathrm{N}$-dimetil- N- bencilamonio de grado técnico.

\section{Prueba de inhibición del yogurt}

Para esta prueba se ensayó la capacidad inhibidora de cada sustancia, como una medida de la especificidad de los inhibidores estudiados (NMX-F-719COFOCALEC-2010). El procedimiento determina el incremento de acidez, expresada en $\mathrm{g} / \mathrm{L}$ de ácido láctico, como resultado de la inoculación de la muestra con un fermento láctico liofilizado: en este caso, el cultivo comercial YO-MIX тм (DANISCO, Francia). Se compara la acidez desarrollada con una leche control negativa preparada a partir de la leche descremada en polvo de calidad microbiológica Difco ${ }^{\mathrm{TM}}$ Skim Milk. La acidez se determina mediante titulación con hidróxido de sodio (NMX-F-719COFOCALEC-2008).

Los inhibidores (antibióticos y quimioterapéuticos) ensayados se seleccionaron entre los de mayor utilización en medicina veterinaria y sus concentraciones permisibles están determinadas por (SADER/SENASICA, 2020) mediante los valores de Límites Máximos de Residuos (LMR), que se actualizan cada año. 
Además de ensayaron los mismos desinfectantes antes mencionados, que fueron igualmente analizados mediante métodos químicos (Tabla 2).

Tabla 2. Inhibidores bacterianos que se ensayaron mediante la prueba de yogurt

\begin{tabular}{ll}
\hline \multicolumn{1}{c}{ Grupo } & \multicolumn{1}{c}{ Compuesto } \\
\hline $\boldsymbol{\beta}$-lactámicos & Ampicilina \\
& Amoxicilina \\
Tetraciclinas & Penicilina G \\
& Tetraciclina \\
& Oxitetraciclina \\
& Sulfamerazina \\
& Sulfamonometoxina \\
Sulfonamidas & Sulfatiazol \\
& Sulfametoxazol \\
& Sulfadimidina \\
Nitrofuranos & Sulfadiazina \\
& Furaltadona \\
Fenicoles & Furazolidona \\
Derivados clorados & Nitrofurazona \\
Compuestos de amonio & Cloranfenicol \\
cuaternario & Hipoclorito de sodio \\
& N-Alquil (C 12,16$)-\mathrm{N}, \mathrm{N}-$ dimetil- N- \\
Oxidantes & bencilamonio \\
\hline
\end{tabular}

Todos los antibióticos y quimioterapéuticos utilizados fueron estándares de grado analítico.

\section{Resultados y discusión}

En la Tabla 3 se presentan los LD de los adulterantes ensayados obtenidos por métodos químicos mientras que en la Tabla 4 se presentan los LD obtenidos con la prueba del yogurt para la cepa comercial YO-MIX ${ }^{\mathrm{TM}}$. En ambas tablas puede apreciarse que la mayor parte de los límites de detección de adulterantes e inhibidores no estaban reportados de forma cuantitativa en la normatividad vigente en México (NOM-243-SSA12010). Teniendo en cuenta la complejidad del abordaje de éste tema, procederemos a hacerlo de manera individual.

Tabla 3. Límites de detección (LD) obtenidos por métodos químicos para los adulterantes ensayados

\begin{tabular}{|c|c|c|c|}
\hline Compuesto & $\begin{array}{c}\text { Límite de } \\
\text { detección (LD) } \\
\text { obtenido }^{+} \\
(\mathrm{mg} / \mathbf{k g})\end{array}$ & $\begin{array}{l}\text { Límite de detección } \\
\text { reportado }\end{array}$ & Referencia \\
\hline Almidón & 125 & \multirow{2}{*}{$500 \mathrm{mg} / \mathrm{kg}$} & \multirow{2}{*}{ CEE, 2008} \\
\hline Fécula de maíz & 250 & & \\
\hline Sacarosa & 10000 & Positivo & Artica, 2016 \\
\hline
\end{tabular}




\begin{tabular}{|c|c|c|c|}
\hline Gelatina & 250 & Positivo & AOAC, 2006 \\
\hline \multicolumn{4}{|c|}{ Agentes oxidantes } \\
\hline Peróxido de hidrógeno & 6 & Positivo & $\begin{array}{l}\text { NOM-243-SSA1- } \\
2010\end{array}$ \\
\hline \multicolumn{4}{|c|}{ Derivados clorados } \\
\hline Hipoclorito de sodio & 25 & Positivo & \\
\hline \multicolumn{4}{|c|}{ Compuestos de amonio cuaternario } \\
\hline Cloruro de benzalconio & 12 & \multirow[b]{2}{*}{$1 *$} & \multirow[b]{2}{*}{$\begin{array}{l}\text { NOM-243-SSA1- } \\
2010\end{array}$} \\
\hline $\begin{array}{l}\mathrm{N}-\text { Alquil }(\mathrm{C} 12,16)- \\
\mathrm{N}, \mathrm{N} \text {-dimetil- N- } \\
\text { bencilamonio }\end{array}$ & 6 & & \\
\hline $\begin{array}{l}\text { ND: No detectable } \\
{ }^{+} \text {Valor obtenido en }\end{array}$ & 195 & ras. & \\
\hline
\end{tabular}

Tabla 4. Límites de detección obtenidos para los inhibidores ensayados con la prueba del yogurt para la cepa comercial YO-MIX ${ }^{\mathrm{TM}}$

\begin{tabular}{|c|c|c|c|}
\hline Compuesto & 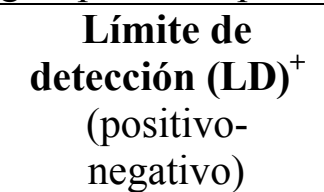 & $\begin{array}{l}\text { Límite } \\
\text { máximo de } \\
\text { Residuo } \\
\text { (LMR) }\end{array}$ & Referencia \\
\hline \multicolumn{4}{|c|}{ Derivados clorados } \\
\hline Hipoclorito de sodio & $25 \mathrm{mg} / \mathrm{kg}$ & ND & NOM-243-SSA1-2010 \\
\hline \multicolumn{4}{|c|}{ Agentes oxidantes } \\
\hline Peróxido de hidrógeno & ND & ND & NOM-243-SSA1-2010 \\
\hline \multicolumn{4}{|c|}{ Compuestos de amonio cuaternario } \\
\hline $\begin{array}{l}\text { Cloruro de } \mathrm{N} \text { - alquil } \\
(\mathrm{C} 12,16)-\mathrm{N}, \mathrm{N}-\text { dimetil- } \\
\text { N-bencilamonio }\end{array}$ & $60 \mathrm{mg} / \mathrm{kg}$ & ND & NOM-243-SSA1-2010 \\
\hline \multicolumn{4}{|c|}{ Antimicrobianos } \\
\hline Tetraciclina & $100-50 \mu \mathrm{g} / \mathrm{L}$ & $100 \mu \mathrm{g} / \mathrm{L}$ & \multirow{13}{*}{$\begin{array}{l}\text { SADER/SENASICA, } \\
2020\end{array}$} \\
\hline Oxitetraciclina & $100-50 \mu \mathrm{g} / \mathrm{L}$ & $100 \mu \mathrm{g} / \mathrm{L}$ & \\
\hline Penicilina $\mathrm{G}$ & 4- $2 \mu \mathrm{g} / \mathrm{L}$ & $4 \mu \mathrm{g} / \mathrm{L}$ & \\
\hline Ampicilina & $10-5 \mu \mathrm{g} / \mathrm{L}$ & $40 \mu \mathrm{g} / \mathrm{L}$ & \\
\hline Sulfadimidina & $20.0-15.0 \mu \mathrm{g} / \mathrm{L}$ & $25 \mu \mathrm{g} / \mathrm{L}$ & \\
\hline Sulfamerazina & $15.0-10.0 \mu \mathrm{g} / \mathrm{L}$ & $100 \mu \mathrm{g} / \mathrm{L}$ & \\
\hline Sulfamonometoxina & $22.0-20.0 \mu \mathrm{g} / \mathrm{L}$ & $100 \mu \mathrm{g} / \mathrm{L}$ & \\
\hline Sulfatiazol & $15.0-10.0 \mu \mathrm{g} / \mathrm{L}$ & $100 \mu \mathrm{g} / \mathrm{L}$ & \\
\hline Sulfadiazina & $10.0-8.0 \mu \mathrm{g} / \mathrm{L}$ & $100 \mu \mathrm{g} / \mathrm{L}$ & \\
\hline Furaltadona & $0.8-0.4 \mu \mathrm{g} / \mathrm{L}$ & ND & \\
\hline Furazolidona & $0.8-0.4 \mu \mathrm{g} / \mathrm{L}$ & ND & \\
\hline Nitrofurazona & $4.0-2.0 \mu \mathrm{g} / \mathrm{L}$ & ND & \\
\hline Cloranfenicol & $2.0-1.0 \mu \mathrm{g} / \mathrm{L}$ & ND & \\
\hline
\end{tabular}

ND: No detectable, límite de tolerancia " 0 "

${ }^{+}$Valor obtenido en al menos el $95 \%$ de las muestras.

Almidón y fécula de maíz 
Para el almidón solamente se encontró el límite de detección del método de prueba para la Comunidad Europea (CEE, 2008). En éste documento se establece que la detección se utiliza como marcador para leche en polvo desnaturalizada, por lo tanto, adulterada.

Ambas sustancias ensayadas tuvieron un límite de detección inferior al límite reportado por la CEE (2008), por lo que dicho método de prueba es válido para el propósito que se describe.

\section{Sacarosa y gelatina}

Estas dos sustancias no son componentes normales de la leche, por lo que no pueden ser consideradas más que adulterantes. El azúcar más importante de la leche es la lactosa, por lo tanto, la presencia de sacarosa en leche sólo puede ser debida a adulteración, que se añade a fin de enmascarar la adulteración con agua (Artica, 2016). No obstante, el límite de detección que se obtuvo para sacarosa en leche fue muy elevado (10 $\mathrm{g} / \mathrm{kg}$ ), lo que lo hace muy poco sensible, $\mathrm{y}$ por lo tanto, de poca utilidad dicha prueba.

Por otra parte, la gelatina, que también podría ser usada para este fin, se detecta a $250 \mathrm{mg} / \mathrm{kg}$, pero sería necesario diluirla previamente antes de incorporarla a la leche, por lo que desde el punto de vista práctico, éste no debería ser un recurso muy utilizado en leche.

En trabajos previos realizados en Jalisco, se encontró una baja incidencia de sacarosa $(2.5 \%$ en leche cruda y $0.7 \%$ en leche pasteurizada); a pesar del elevado límite de detección del método utilizado (Noa et al, 2019), lo que significa que el nivel de adulteración en ese caso era muy alto.
Los LD obtenidos para derivados clorados (hipoclorito de sodio) fue de 25 $\mathrm{mg} / \mathrm{kg}$ utilizando el método químico (Tabla 3), pero éste no fue detectable utilizando la prueba del yogurt (Tabla 4). En este punto, es necesario establecer una definición: la Federación Internacional de Lechería considera, desde 1997, que la presencia en leche de desinfectantes, como los agentes oxidantes (peróxido de hidrógeno), y los derivados clorados (hipoclorito de sodio), sería mínima con la aplicación de buenas prácticas de obtención (menos de $2 \mathrm{mg} / \mathrm{L}$ ). No obstante, exceptuando un mal drenaje del equipo después de la desinfección, la adulteración o adición intencional podría ser la causa de su presencia. Esta mala práctica de manejo se realiza con el fin de obtener una buena calidad bacteriológica (FIL/IDF, 1997) en la leche cruda. En el trabajo de monitoreo antes mencionado (Noa et al, 2019), se encontró $14.7 \%$ de incidencia en leche pasteurizada, específicamente en una marca (83\%) y sólo $0.7 \%$ en leches crudas analizadas, lo que indica la falta de aplicación de las buenas prácticas de manufactura, y no en la obtención de la materia prima.

La prueba de inhibición del yogurt no es específica, por lo que detecta cualquier inhibidor de crecimiento microbiano, incluyendo desinfectantes como los derivados clorados, oxidantes y de amonio cuaternario, pero es mucho más sensible a los residuos de antibióticos, que provienen de un animal bajo tratamiento anti- infeccioso (NOM-243SSA1-2010). Por esa razón, se consideran a los desinfectantes como posibles adulterantes y además como inhibidores. Fue por ello que se decidió, ensayarlos mediante el método de prueba de inhibición del yogurt, además de los métodos de prueba químicos.

Derivados clorados 
En este caso, se estima que los límites de detección de Streptococcus thermophilus y Lactobacillus bulgaricus (3:1) pueden variar dependiendo de la cepa utilizada, por lo que se determinaron siguiendo la recomendación de FIL/IDF (1991). La detección de desinfectantes de las familias de derivados clorados, oxidantes o de amonio cuaternario, a partir de los resultados antes mostrados, no fue lo suficientemente sensible mediante la prueba de yogurt utilizando la cepa YO-MIX TM.

Los límites de detección obtenidos para los antibióticos y quimioterapéuticos ensayados (14 en total) para la cepa comercial YO-MIX TM, se seleccionaron tomando en cuenta mayormente algunos que no aparecían reportados por FIL/IDF (1991). Los valores de LMR se actualizan cada año a partir de 2017, en tanto la NOM-243-SSA1-2010 establece sólo valores cualitativos, y data del 2010.

Los resultados mostrados en la Tabla 4 indican que la cepa de yogurt ensayada permite detectar valores de concentración inferiores o iguales, a los LMR establecidos oficialmente para los antibióticos y quimioterapéuticos ensayados (SADER- SENASICA, 2020), por lo que serían adecuados para detectar, de forma cualitativa la presencia de éstos tipos de residuos en leche, no así la presencia de derivados clorados, oxidantes o compuestos de amonio cuaternario.

\section{Conclusiones}

Los resultados indican que los métodos analíticos aplicados permiten la detección cualitativa de los adulterantes almidón, fécula de maíz y gelatina; mientras que la prueba del yogurt detectó algunos compuestos inhibitorios como los antimicrobianos. Sin embargo, los métodos empleados no fueron lo suficientemente sensibles en ladetección de derivados clorados y compuestos de amonio cuaternario y no detectaron la presencia de sacarosa y agentes oxidantes. Ello implicaría la necesidad de aplicar ambos tipos de métodos: químicos y la Prueba del Yogurt), de forma simultánea, para abordar la presente problemática. De igual manera se pudieron determinar de manera cualitativa los límites de detección de otros adulterantes, no establecidos aun en la normativa mexicana vigente.

La presencia de adulterantes en leche pone en riesgo a los consumidores por lo que se considera un problema de salud pública. La detección y establecimiento de límites de detección de adulterantes presentes en leche son relevantes para el control de calidad ya que, involucra a productores, comercializadores e intermediarios, quienes para producir un alimento de calidad, deben aplicar buenas prácticas a lo largo del proceso productivo.

\section{Literatura citada}

AOAC. Official Methods of Analysis of AOAC International. 18th Edition. Rev. 1. (2006). Edited by: William Horwitz and Latimer, G. W., Gaithersburg, MD. 33.2.37.

Artica, M, L. (2016). Métodos para el análisis fisicoquímico de la leche $\mathrm{y}$ derivados lácteos. Peru, Editorial@ Libros y editoriales, TEIA. 3a. Edición. 146.

CEE (2008): Reglamento (CE) No 273/2008 de la Comisión. Determinación cualitativa del almidón en la leche desnatada en polvo, en la leche en polvo desnaturalizada y en los piensos compuestos. Disponible en https://eur-lex.europa.eu/legalcontent/es/TXT/?uri=CELEX\%3A320 08R0273.

CODEX ALIMENTARIUS. (2017) CXG 90-2017: Directrices sobre criterios de rendimiento para métodos de análisis 
para la determinación de residuos de plaguicidas en los alimentos y los piensos.

FIL/IDF. (1991): Yogurt Inhibitor Test in Bulletin of the International Dairy Federation $\mathrm{N}^{\circ}$ 258/1991: Detection and Confirmation of inhibitors in milk and milk products. $2^{\text {nd }}$ Edition. Brussels (Belgium). 46- 48.

Goodman y Gilman's. (2008). Manual of Pharmacology and Therapeutics. Editors L. L. Brunton, K L. Parker, D K. Blumenthal, I. L.O. Buxton. McGraw- Hill Medical, New York. 707.

LEY GENERAL DE SALUD Nueva Ley publicada en el Diario Oficial de la Federación el 7 de febrero de 1984. TEXTO VIGENTE. Últimas reformas publicadas DOF 24-01-2020 Disponible en http://www.diputados.gob.mx/LeyesBi blio/pdf/142 240120.pdf

NMX-F-700-COFOCALEC-2012.

Norma Mexicana- Sistema producto leche- alimento - lácteo - leche cruda de vaca - Especificaciones Fisicoquímicas, Sanitarias y Métodos de Prueba. Organismo Nacional de Normalización del COFOCALEC. Disponible en http://www.cofocalec.org.mx/catalogo /por clave

NMX-F-719-COFOCALEC-2008.

Norma Mexicana. Sistema- producto leche. Alimentos. Lácteos. Detección de inhibidores bacterianos en lecheMétodos de prueba rápidos. Disponible en http://www.cofocalec.org.mx/catalogo /por clave

Noa, M., Landeros P. Gómez, Z., González, D.G., Real, M., Medina M.S. y Reynoso R. (2019): Incidencia de adulterantes en leches cruda y pasteurizada en el estado de Jalisco, México. e-CUCBA. 6 (12), 15- 28. Disponible en http://ecucba.cucba.udg.mx/index.php/eCucba/article/view/133/126.
Noa-Lima, Elizabeth, Noa, M., González Delia, Landeros, Patricia, Reyes, Waldina. (2009). Evaluación de la presencia de residuos de antibióticos $\quad \mathrm{y}$

quimioterapéuticos en leche en Jalisco, México. Rev. Salud Animal.

$$
\begin{array}{ll}
\text { 31(1): } & 29-33 . \\
\text { Disponible } & \text { en }
\end{array}
$$

http://scielo.sld.cu/scielo.php?script=s ci arttext\&pid=S0253570X2009000100006

NOM-155-SCFI-2012. Norma Oficial Mexicana. Leche-Denominaciones, especificaciones fisicoquímicas, información comercial y métodos de prueba. Disponible en http://www.dof.gob.mx/normasOficial es/4692/seeco/seeco.htm.

NOM-243-SSA1-2010, NORMA Oficial Mexicana Productos y servicios. Leche, fórmula láctea, producto lácteo combinado y derivados lácteos. Disposiciones y especificaciones sanitarias. Métodos de prueba. Disponible en http://dof.gob.mx/normasOficiales/415 6/salud2a/salud2a.htm.

SADER/ SENASICA. Secretaría de Agricultura y Desarrollo Rural/ Servicio Nacional de Sanidad, Inocuidad y Calidad Agroalimentaria. (2020). Límites máximos de residuos tóxicos y contaminantes. TABLA DE LÍMITES MÁXIMOS DE RESIDUOS 2020. Disponible en https://www.gob.mx/senasica/docume ntos/limites-maximos-de-residuostoxicos-y-contaminantes.

Solares, F, A. (2019). Determinación del antibiótico Penicilina en leche de vaca y su repercusión en el rendimiento del procesamiento de queso fresco (Tesis de licenciatura). Centro Universitario de Suroccidente, Universidad de San Carlos de Guatemala. Disponible en http://www.repositorio.usac.edu.gt/12 896/1/TRABAJO\%20DE\%20GRADU ACI\%C3\%93N\%20\%20-\%20ANGELA\%20SOLARES.pdf

Thompson, M., Ellison, S., and Wood, R. (2002): Harmonized Guidelines for 
Single-Laboratory Validation of Methods of Analysis. Pure Appl. Chem. 74(5): 835-855. Disponible en http://publications.iupac.org/pac/2002/ pdf/7405x0835.pdf.

Vega, S., Pérez, N., \& Pinto, M. (1999). Calidad de leche y algunos problemas de adulteración. En: E, Martínez., A, Álvarez., L, García, \& M Del Valle (Coord.) Dinámica del sistema lechero mexicano en el marco regional y global. Editorial Plaza y Valdés. México. 359- 37 